\title{
Accessibility to the Disabled: A Service Oriented Architecture Approach
}

\author{
Ahmed El-Deeb, Member, IEEE \\ Department of Computer Science and Engineering, \\ The American University in Cairo, Egypt. \\ Ahmed.eldeeb@ieee.org
}

\begin{abstract}
As today technology has witnessed remarkable advancement in almost all branches, still much attention needs to be directed to helping those disadvantaged from essential facilities. Specifically, much attention must be paid to the virtual world as it is the main theme of today's technology that makes a big world small. The virtual world is of much help to people with physical impartment. We need not forget those who are in the most need to such technology advancement, the handicapped people. In this paper, I propose a novel solution called "Adaptive Facility Middleware" - a Service-Oriented Architecture-Based middleware- to be incorporated in virtual educational environments enabling the join of handicapped students who wish to earn their degrees despite of their disability. This middleware will not require any virtual learning entity to change its general structure; however, it can be smoothly integrated to the current virtual learning environment to extend its operation to include the handicapped students. The proposed middleware adheres to the Service Oriented Architecture (SOA) which supports the integration of independent services. This makes the middleware extensible and interoperable.
\end{abstract}

\section{INTRODUCTION:}

Nowadays technology is not only advancing in the real world, but it is also advancing in the virtual one opening a mean for innovative globalization that narrows down the world and make it a small village. However, no sufficient efforts have been directed toward people who will directly benefit from the virtual world; they are the physically handicapped people. Today's much efforts to help handicapped people is inclusive to establishing private schools or organization for those people putting them in more alienation from the rest of the world. Those organizations and/or associations yet offer a primarily education enough to get those people able to live a basic life while the rest of healthy people are requested to put pledges to aid those organizations. This makes the physically handicapped people like a burden on the healthy ones while they possess the same mental capabilities and can contribute to the world. What I am trying to stress in this paper is that handicapped people are mentally equal to the healthy ones and can be doctors, engineers, or accountants if they received the proper means to allow them access to professional education. This will not only solve the handicapped own problems, but it will solve our whole world's economical problem by getting those people participate in the advancement of the economy instead of being a burden on it. It will also increase services because those people when they become useful people, they will offer significant services to all kind of people. According to the InfoUse Project funded by the National Institute on Disability and Rehabilitation Research "An estimated $19.4 \%$ of non-institutionalized civilians" in the U.S only have a disability [1]; and it grows.

The proposed system which is called "Adaptive Facility Middleware" is a middleware to be placed between the real physical environment such as a university or a learning center and the virtual handicapped students at their homes or special organizations. The middleware acts as a transformation layer that receives the learning information from one end and converts it to proper representation according to the student's type of disability. The system is interoperable with both the educational environment and the student's environment. Also, the system adheres to the Service Oriented Architecture (SOA) methodology which allows the ability to add or remove independent services without affecting any other interacting parties or involved environments.

\section{RELATED WORKS:}

There have been many movements in the world trying to address the problem of accessibility to disabled people. The Americans with Disabilities Act (ADA) launched at 2000 called for considering disabled people in the web development [2] in its section 508 that disabled people 
shall have access to electronic information and the digital world without discrimination [3]. A considerable flaw to this act is that it proposes ethical guidelines to be followed in the development world to allow access to disabled people. However, abiding by those guidelines and rules is not guaranteed. Study has shown that only 59 percent of universities basic websites are accessible to disabled people [3]. This rate of providing access to disabled people to basic websites infer that the percentage of websites that enable interaction with disabled students for education or knowledge acquisition is far less this number.

Another more technological oriented endeavor has to deal with people of audio disability. The system proposed was called "Polygon Mesh" that uses Water's abstract-muscle based model that detects the facial expressions and jaws movements in order to generate liable audio expressions [4]. The system uses OpenGL C++ in order to simulate the vocal expressions of the disabled. Nevertheless, the proposed system imposes implementation efforts. The implementation of such system needs heavy integration with biomedical engineering and calls for heavy investment in terms of time and research efforts. Also, the seamless integration of such system with an e-learning infrastructure and the reliability of its simulated output are at question.

A notable study was presented at the proceedings of the fifth international ACM conference on Assistive technologies studied some virtual reality systems aiming at modeling educational systems for disabled students allowing them access to education and communication through virtual reality techniques. One studied system that can be used by blind students called "VirtualAurea" that is uses spacial sounds to convey a virtual world to students; for example, by embodying the structure of the classroom and the school map through sounds and it can customized by parents and teachers to accommodate other objects; another system for deaf students called "Mehida" that uses some techniques to enhance communication such as: finger spelling, hand gestures, and lip reading [5].

One major limitation in all systems discussed is that they do not address the difficulty of movement disabled people face. They jump to the conclusion of providing assistive means for the disabled to help them communicate and interact with the world around them; however, they do not address the problem from its early root which is that disabled people face the problem of commuting from one place to another. Another major limitation is the complexity of integrating such technologies with already existing educational online infrastructure. Such proposes systems did not show the handle of interoperability and compatibility issues associated with them.

\section{THE ADAPTIVE FACILITY MIDDLEWARE:}

The proposed middleware as a novel solution to the interaction needed between handicapped people and educational institutions is built on a Service-Oriented Architecture (SOA) methodology to allow the seamless extensibility and both flexible implementation and interoperation. I have adapted the SOA as an architecture to the middleware in order to address the diversity of disability that requires more service elaboration, the expected advancement in virtual reality technologies that has to be accommodated with minimal cost, and to reduce the cost of implementing educational system for the disabled for an online educational institution that want to offer education to the disabled students; hence, encouraging them to help the disabled.

\section{A. $\quad$ SOA as an Architecture:}

The SOA as an architectural organization aiming at treating system components as independent services that uses message passing to communicate with one another, it is extensible because as more services needed to be added later on to upgrade the virtual education system, it can be attached to the middleware easily without reimplementation efforts; components-off-the-shelf (COTS) and web services can be integrated to enhance the service of the middleware. The SOA enables platform independence feature for services implementation. This is a highly desirable feature of a system where learners behind different operating systems can have access to the educational system; thus, assuring accessibility. The implementation independence of programming languages offered by SOA will facilitate more integration and system enhancement as many developers can develop their own services and offer it to the system; it encourages global collaboration in software development to solve the accessibility problem of the disabled people. Services and applications can be integrated as a reusable code which reducing the implementation time and effort while assuring wide accessibility [6].

\section{B. $\quad$ Adaptive Facility Middleware Services:}

The proposed middleware is composed of different services which are used to deliver convenient means for the disabled students to access the virtual e-learning environment and interact with it. Essentially, more and more services can be added and different technologies can be adopted as provided by the SOA.

1. Blind Students: 
The blind students are deprived from the ability to see things. However, they possess high sensitivity to hearing and sensing senses. The learning materials for blind students like books are available for them in Braille's method of writing. Thus, there is no problem for them to have access to hardcopy learning materials. However, what they are deprived from is the direct interaction in the real learning process. They are impaired with two obstacles regarding that. Firstly, there is a mobility problem because blind people have difficulty moving from place to place unless there is a guide which creates a dependency problem. Thanks to virtual technology, today there are e-learning environments provided by many respectable universities worldwide. Secondly, there is an interaction and involvement problem because blind people cannot get involved in the learning activity in classrooms. They will not able to see what's written on the virtual whiteboard or see the virtual handouts distributed in class which is considered a significant impairment especially for students wishing to pursue studies in science and engineering.

The adaptive facility middleware is to be placed between the blind students' environment and the virtual classroom. The middleware is provided with a text-to-speech service which transforms any written material into audio. This includes what's written on the virtual whiteboard by the instructor, all handouts distributed in class time, and assignments. Also, if the lecture entails diagrams or drawings done on the virtual whiteboard, the text-tospeech service can audibly describe the drawing; this can be achieved by incorporating a pattern recognition algorithm that can track the pattern of the drawing and convey it in an audio format. The transformed text into audio is done in real time while the lecture is being conducted to enable the blind students to participate actively in the lecture. Also, they are being recorded to be available for them to download the lectures for their later reference.

\section{Mute Students:}

Mute students are considered a different case from the latter two because they do not have any problem hearing the lecture, viewing the writing, or any learning material. However, they lack expressing themselves. This is crucial for the learning process because students need to interact with the instructor through asking questions or participating in class discussion. This problem can be solved in two techniques one of them is considered to be primitive and other is considered more sophisticated and automated. The first technique is to allow the mute students to type to the instructors in the lecture real time in a chat manner. The instructor and colleagues shall be notified upon receiving a message from the mute students.
The second technique is to provide the mute students with a webcam at their end which will capture snapshots for their hand gestures as they express themselves normally with their sign language. There will be stored pictures of each sign in the sign language database by which the system will compare the taken snapshots of the mute student's hand gestures with the stored pictures in the database; then, displays the equivalent word to the instructor and colleagues in text or audio.

\section{Deaf Students:}

Deaf students on the contrary have the problem of hearing the live lecture conducted by instructors. Basically, deaf students can read lecture notes and study from books with no problem; however, live lectures are very significant for the learning process and hearing the instructors' explanation impact the level of learning for students.

The middleware is provided with a service that translates spoken words into sign language for the deaf people to interpret. As one of the goals of the middleware is to reduce efforts, cost, and easily incorporation of the disabled students, it is not feasible to conduct training courses for instructors in order for them to learn the sign language to teach for the disabled students. Also, we are integrating the disabled to the normal learning facilities; thus, healthy students are existent in the learning environment. Two types of spoken-to-sign-language services can be incorporated in the middleware.

Firstly, the spoken-to-sign-language service can be provided in an algorithm form that takes the audio spoken word as an input, generate corresponding code for it, match it against the sign-equivalent code stored in a database, and display the corresponding sign to the disabled student. This type of service will require a high computational performance in order to carry out the service efficiently although some delay must be considered. This requirement is not problematic since processing technologies are advancing rapidly. A notable advancement is the Intel's announcement published at the ACM TechNews of a new family of microprocessors to be released at the first quarter of 2008; these new chips called "Penryn" are made up of 16 processors to be manufactured in Intel Core 2 and Xeon microprocessors and marked as Intel Core 2 Extreme in notebook PC's [7]. Such example of advancement shall tackle the performance issue of the translating algorithm.

Secondly, another compatible spoken-to-sign-language service that can be incorporated in the middleware is proposed by IBM researchers who developed a system they call SiSi short for "Say-it-Sign-it" that is capable of 
converting spoken language into sign language and can be extended to different languages. The system which uses speech recognition to animate digital character has been approved by the Royal National Institute for Deaf people (RNID) [8]. Such developed system can be seamlessly integrated into the middleware and help deliver functionality to deaf people.

\section{OVERALL ARCHITECTURE \& WORKING:}

the instructor is located. It is also used for pre-recorded lectures posted by the instructor.

\section{B. Adaptive Facility Middleware:}

The Adaptive Facility Middleware consists of a "Text-toSpeech" service component which will be responsible for transforming the text written on the virtual whiteboard, the text of handouts or assignment to audio for the blind

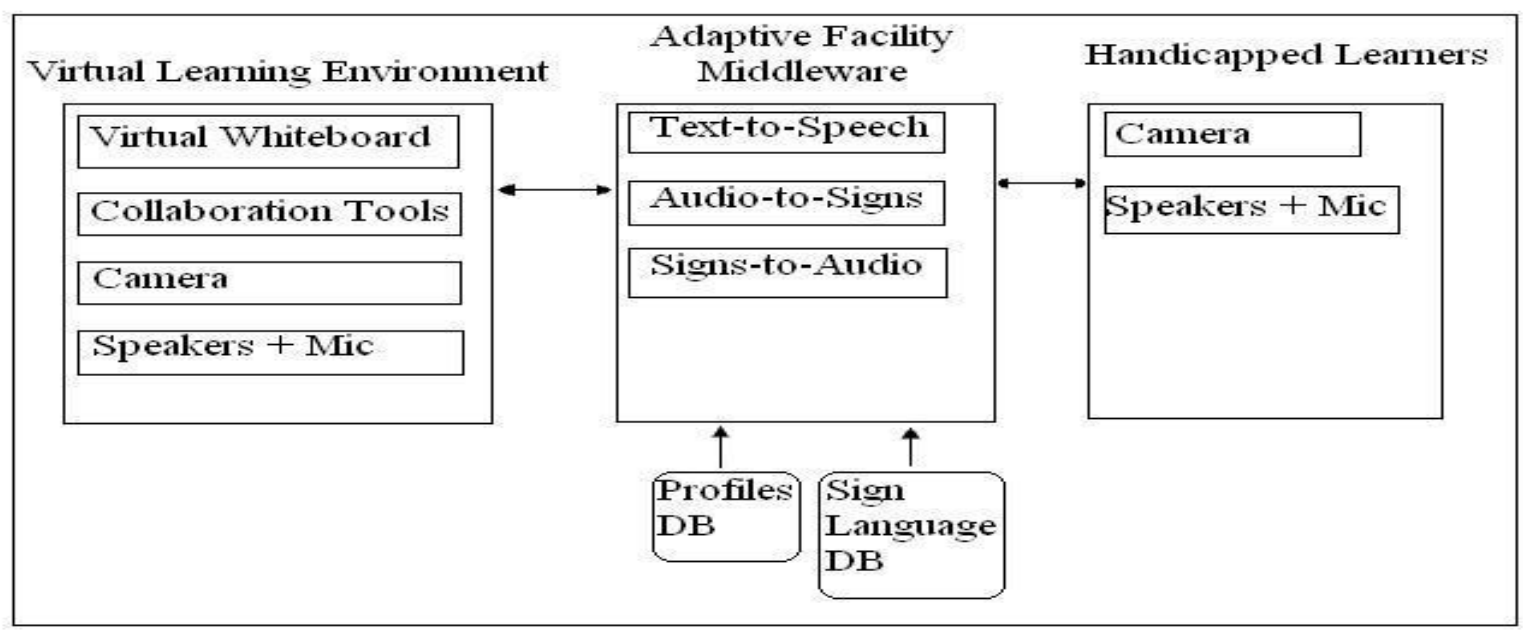

The Adaptive Facility Middleware as shown in the figure is to be placed between the handicapped learners and the virtual classroom conducted at the university. This middleware will be acting as a transformation layer between the virtual learning environment and the handicapped learners by which all learning means shall be transformed into a convenient representation addressing the type of student's disability.

\section{A. $\quad$ Virtual Learning Environment:}

This layer consists of all the e-learning facilities such as the whiteboard which will be used by instructors for conducting a lecture. It will contain also other normal collaboration tools such as a mailing facility, grading system, discussion board. The video component of that layer is to live broadcast the instructor while conducting the lecture. It is also used for recorded tutorials or other videos posted by the instructor. The Audio component is to transmit the instructor's voice while lecturing through a microphone attached to the physical environment where students, an "Audio-to-Sign" service component for deaf students to transform spoken words to deaf sign language, and a "Sign-to-Audio" service component for mute students to transform their sign language as they interact to audio for instructors and colleagues. Those components are explained at the previous section.

\section{i. Profiles Database:}

The middleware is connected to a "profile database" containing all handicapped students profiles. As part of the information written in each student's profile is his/her disability type, upon the student's login to the system using his/her unique username and password the system can check in that database the disability type of the logged students in order to adapt itself dynamically according to the student's need. This allows the system to efficiently and appropriately serve the students.

\section{ii. Sign-Language Database:}


The sign language database is crucial for the involvement of deaf and mute students. It contains two critical forms of data. Firstly, it contains pictures of each sign of the sign language. Secondly, it contains equivalent code for the sign language itself. The system will query to that database in order to match the taken snapshots of the mute students' sign language and also in order to compare the generated code for the spoken words with the equivalent sign code to display the appropriate sign to the deaf students.

The involved two parties of the whole system which are the handicapped students party and the virtual classroom party are interacting with the middleware by providing the necessary input through the attached cameras and microphones and retrieving the services output from the middleware.

\section{CHALLANGES:}

The implementation of the SOA Adaptive Facility Middleware poses a unique research direction that has its own challenges on the conceptual and the implementation level. The conceptual challenge is represented in the extensive research that has to be conducted and the research questions that have to be answered in order to implement a robust system. The implementation challenge is represented mostly in performance issues rather than feasibility issues. The proposed middleware and overall system do not carry any hindrance to the available technologies and science; the question is a about the performance of such middleware. I have tried to address some of the foreseen challenges under those two categories.

\section{A. Conceptual Challenges:}

The implementation of the middleware and such type of a system requires exhausted research in many branches of computing. It involves a data management research in order to decide on how the sign symbols will be stored in a database and to address the question of querying the symbols from the database in the transformation process which has to be efficient. Another important area of research is related to the basic artificial intelligence field regarding the pattern recognition; however, this challenge can be of minor effect since many workarounds can be made other than having an artificial intelligent algorithm.

\section{B. Performance Challenges:}

The most crucial areas of research are on the electronics technology field regarding the processing efficiency and on the algorithm field regarding the algorithm efficiency. Extensive research has to be undertaken in the electronics technology because in order for the middleware to perform efficiently in timely manner, it has to be places on capable machine acting as a server. Although electronics technology is advancing and Intel is expected to penetrate the market with super speed microprocessors, a research is still due in this area. On the other hand, extensive analysis and research must be undertaken regarding the executing algorithms. These performance issues are crucial to such systems because it is an educational system and timely manner response is a must. The system shall aid the education process and shall not exhibit delaying features that may waste the lecture time, cause difficulty to some students to connect, or be relatively slow especially in exams or important lectures.

\section{CONCLUSION:}

This disabled-friendly e-learning application is meant to allow disabled students to have equal access to different fields of learning and from different universities around the world. Not only this system will solve disabled students' educational problems, but it will also solve their psychological problems. Those people feel disadvantaged from the world which causes them constant depression. They will no longer feel the sense of pity from other people because they will be self-independent and will be able to reach high levels of professional education which will allow them to produce and even add new inventions to the world instead of sitting waiting for pledges. They will not try to hide from society as now they will be presented in it as leaders not as a burden and they will have self-worth and will feel a high sense of dignity which will not be present without the aid of such system. The system will also narrow the gap between disabled people and normal ones as they will be equally receiving opportunities. Those people have need for achievement in order to defeat their disability and such system will be the instrument.

\section{FUTURE WORK:}

This middleware sets new research directions as it brings our attention to the marginalized world of disabled students. Disabled people are always put in the last item in our thinking; however, it is due time to start thinking of them because it is our responsibility as healthy educated people to plan and do something useful to help those people. An intensive field of research can be made on two areas that complement each other. One area is the field of e-learning itself; by researching the current technologies 
involved in that trend and whether they are suitable to be adapted or we still need to find other technologies that boost the standard of distant learning as we are entering the era of wireless communication. The other area is the disabled people themselves; we need to research those people's current condition, compare and contrast them with those healthy people or disabled people who had their way in success. We need to research the psychological conditions due to disability especially newly disabled people. We need to investigate and probe their needs and their interpretation of the future; they are full of brilliant ideas. By conducting those two branches of research, we will be holding two solid pieces of information; the tool and the audience of that tool. These will gives us a great opportunity to start doing something for humanity.

\section{REFERENCES:}

[1] InfoUse Project: Access to Disability Data. In: http://www.infouse.com/disabilitydata/disability/1_1.php. 15/10/2007.

[2] Chaomeng James Huang: Usability of E-Government Websites for People with Disabilities. In: the proceedings of the $36^{\text {th }}$ Hawaii International Conference on System Sciences. IEEE Computer Society, 2002.

[3] Eleanor T. Loiacono, Scott McCoy, William Chin: Federal Web Site Accessibility for People with Disabilities. In: IT Pro. IEEE Computer Society, 2005.
[4] JESULIANA NASCIMENTO ULYSSES, AURA CONCI: A Speech Orientation Animation System to Disabled People. In: the proceeding of the XV Brazilian Symposium on Computer Graphics and Image Processing. IEEE Computer Society, 2002.

[5] Jaime Sanchez, Nelson Baloian, Wolfram Luther: Modeling Educational Software for People with Disabilities: Theory and Practice. In: the proceedings of the fifth international ACM conference on Assistive technologies, 2002.

[6] Christophe Schroth: Web 2.0 versus SOA: Converging Concepts Enabling Seamless Cross-Organizational Collaboration. In: the $9^{\text {th }}$ International Conference on ECommerce Technology and the $4^{\text {th }}$ International Conference on Enterprise Computing, E-Commerce and E-Services. IEEE Computer Society, 2007.

[7] John Markoff: Intel to Unveil Chips for Improving Video Quality on the Web. ACM TechNews: the New York Times Technology, Nov. 2007.

[8] Geoff Adams-Spink: Technique Links Words to Signing. ACM TechNews: BBC Technology News, Sep. 2007. 